\title{
Effect of FGF/FGFR Signal in Fluid Shear Stress and Estrogen Regulating Bone Metabolism
}

\author{
Xia-Yan Luo ${ }^{1 \#}$, Yun Yang ${ }^{1 \#}$ and Hui-Qiang Sun ${ }^{1,2^{*}}$ \\ ${ }^{1}$ Department of Prosthodontics, College of Stomatology, Shandong University, China \\ ${ }^{2}$ Shandong Provincial Key Laboratory of Oral Biomedicine, Shandong Province, China \\ \#Authors contributed equally to the work
}

"Corresponding author: Sun HQ, Department of Prosthodontics, College of Stomatology, Shandong University, 44-1 wenhuaxilu Street, Jinan, Shaondong, 250012, China, Tel: +86 0531 88382058; E-mail: whitedove69@163.com

Received date: January 17, 2018; Accepted date: January 25, 2018; Published date: February 01, 2018

Copyright: (c) 2018 Luo XY, et al. This is an open-access article distributed under the terms of the Creative Commons Attribution License, which permits unrestricted use, distribution, and reproduction in any medium, provided the original author and source are credited.

\begin{abstract}
Estrogen and fluid shear stress (FSS) play an important role in bone metabolism, displaying a synergistic effect. Fibroblast growth factors (FGFs) are also of great importance in bone development before and after birth. FGFs regulate downstream signaling pathway by activating FGF receptors (FGFRs) to control the expression of bone tissue cells. This paper conducts a brief discussion of the effect of FGF/FGFR as well as fluid shear stress and estrogen in bone metabolism regulation.
\end{abstract}

Keywords: FGF; FGFR; Estrogen; Fluid shear stress; Bone metabolism

\section{Introduction}

Bone is a kind of tissue that is always in constant dynamic development. Bone metabolism depends upon balanced osteoblast osteogenesis ability and osteoclast bone resorption ability, which are subject to the influence of many factors, such as cytokines, mechanical load and hormone [1]. Estrogen can enhance osteogenesis [2], because it has the dual effect of hindering osteoclast bone resorption and promoting osteoblast osteogenesis [3,4]. Mechanical stress serves as another important factor influencing bone remodeling, for suitable mechanical stress can promote the growth of bone tissues. Cells are more sensitive to FSS than to pressure and stretch stress [5]. Flowing fluid forms FSS on the surface of bone tissue cells, such as osteocytes and osteoblasts. At present, the mechanism of FSS being turned into biochemical signal and introduced into nucleus through the surface of cytomembrane is regarded as stress transduction mechanism [6]. FGFs fall into the family of polypeptides, which control several crucial cellular processes. In bones, FGF/FGFR signals are important regulators for prenatal and postnatal bone development $[7,8]$. This paper mainly discusses the influence of the separate and joint effect of FGF/FGFR signals, estrogen and FSS upon bone metabolism as well as the effect of FGF/FGFR signals in FSS and estrogen regulating bone metabolism. Starting from the intracellular signal paths induced by FGF/FGFR signals, estrogen and FSS, this paper explores their interaction at molecular level, presenting a new approach for following explorations into bone regeneration in clinics and research.

\section{Introduction of the Structures of FGF/FGFR, Estrogen and Estrogen Receptor}

There are two types of FGFs, namely secretion type and intracellular type. Secretion type FGFs can be further divided into canonical type and endocrine type, in which the former contains five subfamilies, namely FGF1, FGF4, FGF7, FGF8 and FGF9, while the latter contains three members, namely FGF15/19, FGF21 and FGF23 (Table 1).

Secretion type FGFs mainly combine with FGFR as signal protein to transmit signals and activate multiple cellular pathways. Intracellular FGF is a kind of non-signal protein, mainly used as voltage-gated sodium channel and the cofactor of other molecules [9-11] (Figure 1).

\begin{tabular}{|c|c|c|c|c|c|c|}
\hline FGF Subfamily & FGF1 Subfamily & FGF4 Subfamily & FGF7 Subfamily & FGF8 Subfamily & FGF9 Subfamily & FGF15/19 Subfamily \\
\hline \multirow[t]{3}{*}{ FGF } & FGF1 & FGF4 & FGF3 & FGF8 & FGF9 & FGF15/19 \\
\hline & FGF2 & FGF5 & FGF7 & FGF17 & FGF16 & FGF21 \\
\hline & & FGF6 & FGF10 & FGF18 & FGF20 & FGF23 \\
\hline
\end{tabular}

Table 1: The subfamilies of FGF. 
Page 2 of 8

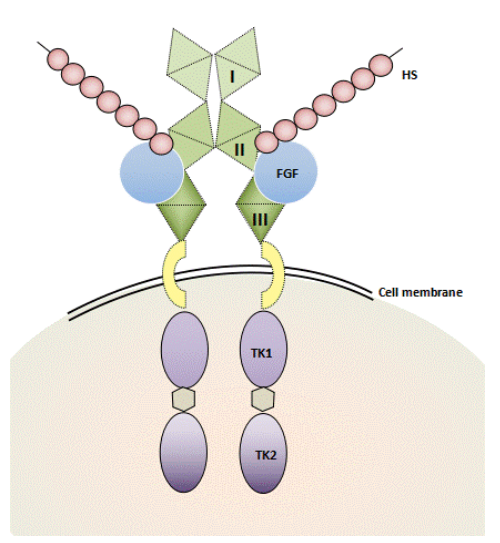

Figure 1: FGF-FGFR structure. (There are two types of FGF family, namely secretion type and intracellular type. FGFR family includes FGFR1, FGFR2, FGFR3 and FGFR4, divided into extracellular, cell membrane and intracellular parts. There are three types of immune globulin structural domains (I, II, III) in extracellular part, one type of transmembrane domain in cell membrane, and two types of tyrosinekinase in intracellular part (TK1, TK2). Structural domain II III and the connexin in between is the binding region of FGF. The specificity of the structure regulates the ligand binding specificity and affinity $[9,13,14]$. FGF and FGFR binding also needs the participation of cofactors to enhance the bioactivity of FGF. HS and HSPG are currently regarded as the potent cofactors of canonical type FGF signaling pathway [15-17]. The cofactor of endocrine type FGFs is Klotho family $[19,20])$.

FGFR is a kind of tyrosinekinase receptor, and shares similar structure with other types of tyrosine kinase receptors, mainly consisting of ligand-binding domain, transmembrane domain and cytoplasmic domain [12-14]. FGFR family includes FGFR1, FGFR2, FGFR3 and FGFR4. Fgfr1-Fgfr3 generates two additional major splice variants of immunoglobulin-like domain III, referred to as IIIb and IIIc. Data is derived from receptor activation assays using BaF3 cells, L6 myoblasts or HEK293 cells transfected with individual splice variants of FGFRs or by direct binding studies [9] (Table 2).

\begin{tabular}{|c|c|c|c|}
\hline FGFR & $\begin{array}{l}\text { FGFR } \\
\text { subfamily }\end{array}$ & FGF & FGF subfamily \\
\hline \multirow[b]{7}{*}{ FGFR1 } & \multirow[b]{2}{*}{ FGFR1b } & FGF12 & FGF1 subfamily \\
\hline & & FGF371022 & FGF7 subfamily \\
\hline & \multirow[b]{5}{*}{ FGFR1c } & FGF12 & FGF1 subfamily \\
\hline & & FGF456 & FGF4 subfamily \\
\hline & & FGF81718 & FGF8 subfamily \\
\hline & & FGF91620 & FGF9 subfamily \\
\hline & & FGF15/192123 & FGF15/19 subfamily \\
\hline \multirow[b]{3}{*}{ FGFR2 } & \multirow[b]{2}{*}{ FGFR2b } & FGF1 & FGF1 subfamily \\
\hline & & FGF371022 & FGF7 subfamily \\
\hline & FGFR2c & FGF12 & FGF1 subfamily \\
\hline
\end{tabular}

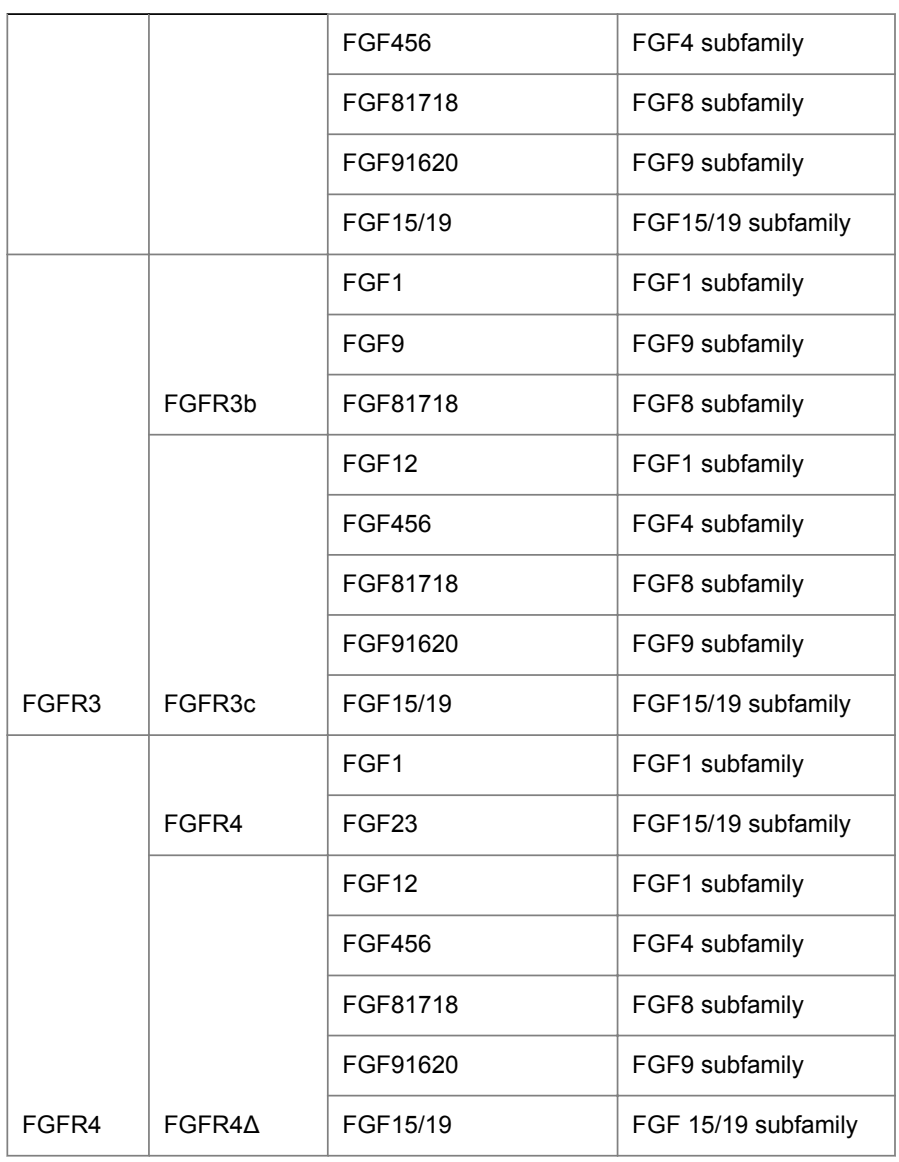

Table 2: Receptor specificity of canonical and endocrine FGFs. (Data is derived from receptor activation assays using BaF3 cells, L6 myoblasts or HEK293 cells transfected with individual splice variants of FGFRs or by direct binding studies [9]).

There are three important processes for FGFR activation, i.e., ligand binding, receptor ligand dimerization and intracellular phosphorylation of receptors [15]. The binding of FGF and FGFR also needs the participation of cofactors to enhance the bioactivity of FGF. Heparan sulfate (HS) and heparan sulfate proteoglycan (HSPG) are currently regarded as potent cofactors for canonical FGF signaling pathways [9,16-18]. The cofactor of endocrine FGFs is a kind of Klotho protein used to form FGF-FGFR-Klotho trimer [19,20] (Figure 1).

Estrogen is an important type of endogenous hormones in human physiology, including estrone (E), 17-estrodiol (E1), estriol (E3), etc. They are mainly produced in ovary and testis and scattered in all cells [21]. Estrogen features extremely important biological functions, including its significant regulatory effect on the metabolism of multiple organs like reproductive system, bone and cartilage system, and cardiocerebral vascular system $[21,22]$.

There are two subtypes of estrogen receptor (ER), namely ERa and ER $\beta$, both of which fall into nuclear receptor hormone superfamily receptor and share common structural framework, i.e., $\mathrm{N}$ terminal (I region), DNA binding region (II region) and $\mathrm{C}$ terminal ligand binding region (III region) [23-27]. Canonical ER pathway is the binding between estrogen and ER, inducing comformational changes of receptors and ultimately leading to homodimerization or heterodimerization $(\mathrm{ER} \alpha-\mathrm{ER} \beta, \mathrm{ER} \beta-\mathrm{ER} \beta$ or $\mathrm{ER} \alpha-\mathrm{ER} \beta)$. The 
homodimerization or heterodimerization generated are further to be transferred to nucleus to make receptor and chromatin in high affinity combined, thereby regulating the transcription of target genes [28-30] (Figure 2).

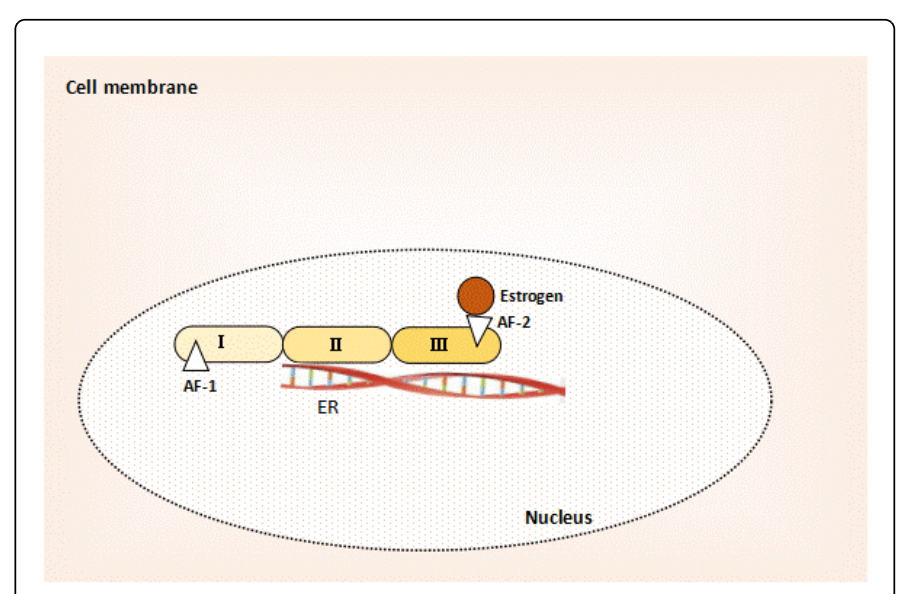

Figure 2: Estrogen-ER structure. (There are two subtypes of ER, namely ER $\alpha$ and ER $\beta$, both of which fall into nuclear receptor hormone superfamily receptor and share common structural framework, i.e., I $\mathrm{N}$ terminal, II DNA binding region and III C terminal ligand binding region [23-25]. In I region, there is one active functional region-AF-1 of ER that does not depend upon ligand, participating in regulating the binding between ER and ligand and response gene transcription and containing a structural domain that could be recognized by estrogen agonist [26]. In III region, there is another active functional region AF-2, with which the ligand can also realize binding [27]).

There is ER expression in many cells, mainly distributed in uterus, ovary, mammary gland, bone tissue, urinary organs, neurocyte and immunocyte, liver and fat, etc. [23]. ER activation is mainly related to phosphorylation [31], in which there are many types of enzyme involved, including tyrosine kinase (TK), casein kinase (CK), etc. [32]. In addition, other signal systems like commonly-seen cell phosphorylases (PKA, PKC), intracellular signals like polypeptide growth factor, kinase, neurotransmitter and regulatory factor can also realize ER phosphorylation [31]. ER serves as an operation node for estrogen to play its role in biological activity inside cells, mainly displayed as cell growth, differentiation, aging and apoptosis $[33,34]$. There is expression of ER $\alpha$ and ER $\beta$ in bone tissues yet with difference in distribution. In cortical bone, ERa is the main subtype expressed, while in bone trabecula it is ER $\beta$ [35]. The activity and number of ER change with the level of estrogen, while with the increase of age or after menopause among females, the expression of ER drops [36].

\section{Estrogen and FSS Regulating Bone Metabolism}

Estrogen is one type of steroids with wide biological activity that balance the osteoblast osteogenesis ability and osteoclast bone resorption ability [22]. Lack of estrogen is prone to cause postmenopausal osteoporosis (OP) [36,37]. In 1941, Albright first adopted estrogen substitution therapy to treat OP [38]. Lack of estrogen may promote the resorption of experimental periodontitis alveolar bone. Estrogen substitution therapy can effectively prevent decreased alveolar bone height and lower the risk of agomphiasis [39]. Research has demonstrated that the icariin separated from epimedium can prevent loss of bone mass, for it can enhance the activity of ERK and JNK in MC3T3-E1 osteoblast yet without obvious influence upon P38. After estrogen receptor antagonist ICI182780 is applied, the division and mineralization abilities of MC3T3-E1 osteoblasts significantly drop, and the phosphorylation of ERK and JNK is also hindered. Therefore, it is deducted that estrogen-ER-ERK/JNK pathway can promote the division and mineralization of osteoblasts and ultimately osteogenesis [40]. Research has proved it is by estrogenER-ERK-Target pathway that estrogen promotes the autophagy of osteoblasts to hinder its apoptosis and ultimately facilitate osteogenesis [41].

FSS stimulated osteoblast proliferation and differentiated stress transduction mechanism involve multiple cell-dependent and independent signaling pathways, such as intracellular and extracellular Ca current [42,43], NOS-NO [44], Cox-PGI2-PGE2, PTX-Gi/o, etc. [6]. Many of them are involved activating one or more members of the MAPK family, including ERK1/2, JNK, P38 and/or interaction with integrin signaling pathway [6]. Integrin, an ECM-cell surface receptor, is connected with cytoskeleton and extracellular matrix, serving as an important mechanical transducer for FSS stimulation and being able to act as mechanoreceptors [45]. ERK1/2 can be activated through integrin/FAK pathway [46]. Research has shown that ERK5 participates in the early reaction of osteoblast against FSS and annular FSS promotes MC3T3-E1 osteoblast proliferation through ERK5/ AP-1/cyclin D1 pathway $[46,47]$. Zhao et al. [48] in the experiment of adding FSS into MC3T3-E1 cells obtained the conclusion that MEK5/ ERK5 pathway could regulate FSS induced osteoblast differentiation. It was reported that FSS working on human osteogenesis sample MG63 cells and MG63 cells could enhance the phosphorylation level of ERK and JNK [49]. Li et al. [50] also proved that FSS could uplift the phosphorylation level of ERK, JNK and P38. Moreover, much research found out that mechanical stimulation could enhance the expression of insulin-like growth factor (IGF), a reaction factor regulating osteogenesis with mechanical load [51,52]. Kahlert et al. [53] further proposed that IGF-ER signaling pathway needed the participation of ERK signal; ERa signal was at the upper stream and downstream of ERK signal and ER was activated through ligand binding with ERK1/2. In addition, signaling pathways like Wnt and Smad also played a crucial role in FSS induced osteogenesis [50,54]. There was a series of research highlighting the importance of $\mathrm{Lrp} 5 / \mathrm{Wnt} / \mathrm{b}$-catenin pathway in loading osteogenesis reaction $[55,56]$, the process of which needs ER [57-59]. Lrp5/Wnt/b-catenin pathway and IGF-ER signal were crosslinked through GSK-3b [44,60].

Many studies have demonstrated the importance of estrogen receptor in GSS-induced osteogenesis [57,61-65]. The increased number of ER could enhance stress or estrogen-related signaling pathway transduction, and all these responses could be prevented by ER antaognists ICI 182, 780, hence proving that these pathways were ER mediated [61]. Research by Lee et al. [62] showed that when mechanical stress was imposed upon the ulna of mice after ERa knockout, the formation amount of was only $1 / 3$ of the newborn mice at the same litter where ERa existed; and the osteoblast extracted from mice after ERa knockout had a weak response to mechanical stress [57]. Damien et al. [63] pointed out that among males and females, mechanical stress could promote osteoblast proliferation through ER. If ROS $17 / 2.8$ cell lineage is exposed to estrogen for $5 \mathrm{~min}$ or monocyclic dynamic strain force was imposed for $5 \mathrm{~min}$, it can be observed that both ERa phosphorylation and ERK1 activation increase, which however can be hindered by MAPK inhibitor U0126 and protein kinase A (PKA) inhibitor PKI. That is to say, osteocyte can 
respond to estrogen or FSS through Estrogen/FSS-ERa-ERK1 pathways $[64,65]$.

Joldersma et al. [66] found that estrogen could enhance the generation of E2 (PGE2) in postmenopausal non-osteoporosis female osteoblasts induced by FSS. Bakker et al. [67] demonstrated the adjection of estrogen and FSS against the PGE2 and NO in osteocytes among females with osteoporosis (age 62-90). Yeh et al. [68] proved that estrogen could enhance FSS, strengthening the phosphorylation of human osteogenesis sample cells (MG63 cells), primary osteoblasts ERK and P38 and increasing the expression of c-fos and Cox-2. This process was realized by estrogen receptor increasing $\beta 1$ integrin. These results suggest that estrogen may influence the response function of osteocytes to FSS and there is a synergistic effect between estrogen and FSS in osteogenesis. Li et al. [50] discovered that estrogen and FSS both had the effect of promoting osteoblast proliferation differentiation and their synergistic effect superseded the independent effect of individual, so they had the synergistic effect (Figure 3).

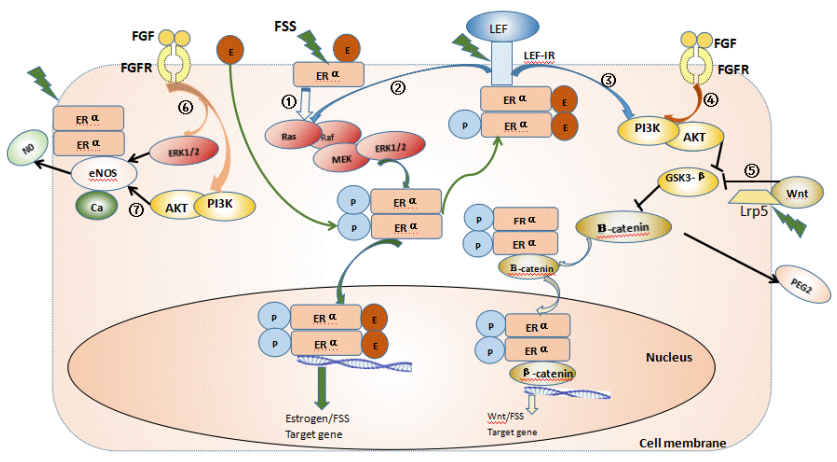

Figure 3: Cross-linking of FSS, estrogen, FGF-FGFR, Wnt- $\beta$-catenin signaling pathways. (1: FGF/FGFR, Estrogen+ER, FSS+ER can directly induce the activation of Ras/Raf-MAPK-ERK1/2 pathway $[40,41,64,68,70,71] .2$ : The activation of IGF-1+IGF-IR+ER induced downstream factors also needs the participation of ERK1/2 signals[51,53]. 3: The effect of ERa and LEF/LEF-1R can also be realized by down-regulating the GSK3beta activity through PI3KAKT [44,51]. 4: FGF/FGFR signal can be transduced to frs2-grb2gab1-PI3K-AKT signal cascade, and down-regulate GSK3beta activity under the condition of being accompanied by Ser 9 phosphorylation [60]. 5: Wnt signal transduces through frizzled receptor and lrp5/6 co-receptor to down-regulate the activity of GSK- $\beta$ (GSK3B) [60]. 6,7: ERK1/2 and PI3K-AKT signals at the downstream of FGF/FGFR also participate in the release of NO [9,44]. 1,2: Realize expression by E-ER binding target gene after entering nucleus. 3,4,5: Influence the $\beta$-catenin-ER nuclear accumulation through regulating the activity of $\beta$-catenin and ultimately regulate the expression of target gene $[28-30,51,58-60,65])$.

\section{FGF/FGFR Signal Regulating Bone Metabolism}

FGF/FGFR signaling pathways have an important effect upon regulating osteogenesis. Much research has proved that FGF/FGFR can promote the division and differentiation of osteoblasts. There are three important processes in FGFR activation, namely ligand binding, receptor ligand dimerization and receptor intracellular domain phosphorylation $[13,67]$. FGF/FGFR signaling pathways start from the extracellular binding of FGF and FGFR and take HS/HSPGs/Klotho as the co-factor to form FGF-HS/HSPGs/Klotho-FGFR 1:1:1 compound; and its dimerization induces juxtaposition and activation of tyrosine kinase structural domain inside FGFR (TK1, TK2) and ultimately activates four types of main intracellular signaling pathways, including RAS-MAPK [12,69-71], PI3K-AKT [70,72,73], PLC $\gamma$ [69] and STAT [74]. The most familiar MAPKs are ERK1/2, JNK, P38 [9,75-78] (Figure 4).

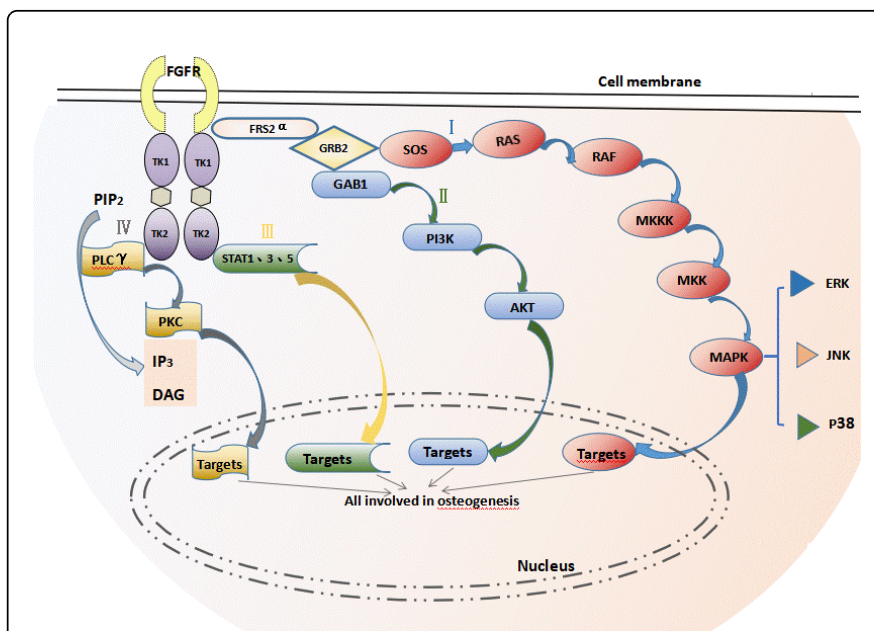

Figure 4: FGF-FGFR signaling pathways. (FGF/FGFR signaling pathways start from the extracellular binding of FGF and FGFR and take HS/HSPGs/Klotho as the co-factor to form FGF-HS/HSPGs/ Klotho-FGFR 1:1:1 compound; and its dimerization induces juxtaposition and activation of tyrosine kinase structural domain inside FGFR (TK1, TK2) and ultimately activates four types of main intracellular signaling pathways, including I RAS-MAPK [69-71], II PI3K-AKT [70,72,73], III PLC $\gamma$ [74] and IV STAT [69]. The most familiar MAPKs are ERK (1/2), JNK, P38, which can be divided into different subfamilies according to genetic coding, namely ERK (1/2), JNK (1/3) and P38 ( $\alpha, \beta, \gamma$ and $\delta$ ) [75-78]. After activation, FRS2 $\alpha$ binds with cytomembrane anchor adaptor protein GRB2 and tyrosine phosphatase SHP2 and then GRB2 collects SOS to activate RAS-MAPK signaling pathway, and collects GAB1 to activate PI3KAKT signaling pathway. FGFR tyrosine kinase activation promotes PLC $\gamma$ phosphorylation, hence causing PI45-HEDP hydrolyzation into IP3 and DAG. IP3 can uplift the level of intracellular Ca ions while DAG can activate PKC protein kinase C [9]).

Recent data showed that FGF2 activated PKC could activate the activity of Runx 2 in mesenchymal stem cells and osteoblasts $[79,80]$. ERK1/2 signals induced by FGF2 were found to promote the proliferation of osteoblast precursor cells and induce FGFR2 induced osteoblast differentiation [70,71]. PLC/PKC activation was also involved in FGF2 or FGFR2 activated osteoblast differentiation [79]. PI3K is another FGF mediated signal cascade reaction controlled by osteoblast cytopoiesis. For example, FGF or FGF4 activated PI3K-Akt pathway promoted the proliferation of osteoblast precursor cells [81]. FGF18-FGFR1/FGFR4 mediated signaling pathways could regulate the differentiation of osteogenesis $[82,83]$. Delayed ossification has been described in FGF18-deficient mice [84], suggesting that FGF18 controls osteogenesis. Recent data indicated that FGF18 played an important role in osteogenesis of mice [85]. The mice mesenchymal stem cells osteoblast differentiation mediated by FGF18 and induced 
by FGFR1/FGFR2 was also involved in PI3K and ERK1/2 signals [83] and the Akt activity increase caused by FGFR2 mutation could promote osteocyte differentiation [73,84,85]. FGF23/FGFR1 signaling pathways were related to bone mass and bone mineralization $[86,87]$.

FGFRs mutation could cause two types of skeleton diseases, namely skeletal dysplasia and craniosynostosis. FGFR1 expression went throughout the bone development and postnatal bone reconstruction, fracture healing and osteogenesis in embryo. Missense mutation of FGFR1 (P252R) could cause Pfeiffer Syndrome [88]. Mice with important fragmentary mutation of FGFR2 scarcity or FGFR2 displayed bone hyperplasia drop and bone mineral density drop. And the absence of FGFR2 in mice could lead to dysfunction of mature osteoblasts [89,90]. Research showed that activated FGFR2 mutation could promote osteoblast differentiation and ultimately cause human craniocerebral disease through increasing Runx2 expression and osteogenic marker genes [91]. Through activated FGFR2 mutation, activated ERK-MAPK could increase Runx 2 transcription activity and osteogenic marker gene expression [81]. Therefore, activated FGFR2 mutation could promote osteoblast differentiation of mesenchymal stem cells [80]. FGFR3 failure and FGFR3 activation both could lead to osteoporosis [92,93]. In mouse model with achondroplasia, chondrocyte specific FGFR3 activation was related to the increase of osteogenesis around cartilage, indicating partial osteogenesis increase [94], which might be indirectly mediated through BMP.

FGF/FGFR signal controlling osteogenesis involved multiple signaling pathway activation. FGF/FGFR signal and canonical Wnt/ $\beta$ catenin pathway had complicated interaction. The loss of efficiency of Wnt inhibitor Axin2 could lead to craniosynostosis of mice $[95,96]$. Experiments of Liu et al. [95] showed that lack of Axin2 could promote $\beta$-catenin signal and ultimately cause ossification of periosteum. The interaction between Wnt and FGF signals activated Wnt signals, mesenchymal stem cell osteoblast differentiation and osteogenesis [97]. Runx2 and Sox2 also played an important role in FGF and Wnt signaling pathway crosstalk $[23,98]$. FGFR1, FGF4, FGF18 and FGF20 were direct target factors of Wnt [97-101].

Wnt-dependent BMP signals could guide intracellular $\beta$-catenin distribution, featuring an important positive feedback regulation effect upon $\beta$-catenin [96]. FGF signal and BMP signal, apart from the Wnt/ $\beta$-catenin mediated indirect relations, also shared certain direct relations. FGF2 could lower the resistance of BMP to noggin and thereby promote BMP signal transduction in skull development [102]. In addition, FGF18 hindered the expression of noggin, hence increasing the BMP signals in cytoskeletons [103]. Endogenous FGF signals up-regulated BMP2 in osteoblasts and skull, and uplifted BMP2 induced ectopia osteogenesis in mice [104-106]. These studies highlighted the positive interaction of FGF and BMP signals in controlling osteogenesis.

\section{Interaction of FGF/FGFR Signals with FSS and Estrogen Regulating Bone Metabolism}

Li et al. [50] after imposing estrogen, FSS or two stimulation factors upon MC3T3-E1 cells, found out that there was obvious change in FGFR1 expression, suggesting that FGFR1 played an important role in regulating the process of estrogen and FSS promoting osteocyte proliferation and differentiation. Further experiments, after intervention of estrogen and FSS upon MC3T3-E1 osteoblasts, saw significant increase of FGFR1 expression. Applying FGFR1 inhibitor PD166866+estrogen+FSS and comparing with control group, the division and differentiation abilities of osteoblasts were hindered, indicating that FGFR1 played a core role in osteoblast response to estrogen and FSS. When estrogen and FSS independently or jointly worked on osteoblasts, the phosphorylation level of ERK, JNK and P38 significantly improved. After applying FGFR1 inhibitor PD166866, the phosphorylation level of ERK was hindered, i.e., FGFR1-ERK signals played a key role in osteogenesis induced by estrogen and FSS. This process was the result of the intracellular signal cascading of FGFR, estrogen and FSS. It remains to be further studied whether estrogen and FSS directly work on FGFR.

It could be obtained from the above discussions of intracellular signaling pathways induced by FGF/FGFR signals, estrogen and FSS that MAPKs-ERK1/2 signaling pathway, Wnt/ $\beta$-catenin signaling pathway, PI3K-AKT signaling pathway and TGF $\beta$-Smads-BMP signaling pathway were cross-linked pathway of the three (Figure 3).

\section{ERK1/2 pathway}

FGF/FGFR, Estrogen/ER and FSS+ER could directly transduce Ras/ Raf-MAPK-ERK1/2 pathway activation $[40,41,64,68,71,72]$. FSS induced NO release [44] and IGF-1+IGF-IR+ER induced downstream factor activation also needed the participation of ERK1/2 signals $[51,53]$.

\section{Wnt/ $\beta$-catenin signaling pathway}

Wnt signals could conduct transduction through frizzled receptor and lrp 5/6 co-receptor to down-regulate GSK3- $\beta$ (GSK3B) activity. FGF/FGFR signals, after transduced to frs2-grb2-gab1-PI3K-AKT signal cascading, also down-regulated GSK3beta activity under the condition of being accompanied by Ser 9 phosphorylation [60]. ERa and LEF/LEF-1R could also down-regulate GSK3beta activity through PI3K-AKT [51,52]. GSK3beta downregulation caused $\beta$-catenin accumulation [60]. FSS could work upon the Wnt/lrp5 on cytomembrane, causing $\mathrm{Wnt} / \beta$-catenin signaling pathway activation; but in this process $\beta$-catenin nuclear accumulation needed ER $\alpha$ mediation $[56,65,68,107]$. The activity and number of ER $\alpha$ changed with the level of estrogen [36], i.e., estrogen could indirectly participate in $\mathrm{Wnt} / \beta$-catenin signaling pathways.

FGF/FGFR signal scan could promote Runx2 activity, while Runx2 could induce FGFR mutation, leading to increased expression of Sox2. The interaction between Sox 2 and $\beta$-catenin hindered the activity of Wnt responsive plasmids and leaded to the downregulated expression of Wnt target genes [98]. And Wnt signals could also hinder Runx2 activity through P38 and ERK signals [23]. In addition, FGF genes are also the direct target genes of Wnt signals [96,99-101].

\section{TGF $\beta$-Smad-BMP signaling pathway}

TGF $\beta$-Smad-BMP signaling pathway and Wnt $/ \beta$-catenin signaling pathway had a synergistic effect, so that those which could work on Wnt signals could indirectly work on TGF $\beta$-Smad-BMP signals [96]. In addition, FGF/FGFR also had a direct effect on TGF $\beta$-Smad-BMP signaling pathway, and Noggin had a negative feedback regulation effect on BMP signaling pathways. FGF18 hindered the expression of noggin, hence increasing the BMP signals in cytoskeletons [102,103]. Meanwhile, Smads could also be activated by P38 and ERK signaling pathways [108] and P38 and ERK were also downstream factors of FGF/FGFR [9]. 


\section{PI3K-AKT signaling pathway}

PI3K-AKT pathway was one of the four canonical pathways for FGF/FGFR signals [9]. FSS worked upon cells to produce NO needed PI3K-AKT signals and ER participation [44]. FSS-IGF-1/IGF-IR/ER [51,53] pathways and FGF/FGFR-PI3K-AKT [60] downstream could down-regulate GSK3- $\beta$ (GSK3B) activity, thereby interacting with $\mathrm{Wnt} / \beta$-catenin signaling pathways.

Based on the above, a great deal of research has demonstrated that the independent effect of FGF/FGFR, estrogen and FSS and the joint effect of estrogen and FSS play an important role in bone development. In addition, this paper also discusses at the level of molecule that MAPKs-ERK1/2 signaling pathway, Wnt $/ \beta$-catenin pathway, PI3KAKT signaling pathway and TGF $\beta$-Smads-BMP signaling pathway are the cross-linked pathways of FGF/FGFR, estrogen and FSS. There is also research shown that FGFR1-ERK signals play a key role in FSS and estrogen induced osteogenesis. In addition, it remains to be studied whether there are other direct or indirect relations between FGF/FGFR, estrogen and FSS.

\section{Acknowledgement}

This work was supported by the Natural Science Foundation of Shandong Province (No: ZR2014HM053).

\section{References}

1. Harada S, Rodan GA (2003) Control of osteoblast function and regulation of bone mass. Nature 423: 349-355.

2. Samuels A, Perry MJ, Tobias JH (1999) High-dose estrogen induces de novo medullary bone formation in female mice. J Bone Miner Res 14: 178-186.

3. Mone Z, Baljit SM, Li S (2003) Understanding osteoblast formation and function: Implications for future therapies for osteoporosis. Curr Opin Orthop 14: 341-350.

4. Nakamura M, Udagawa N, Matsuura S, Mogi M, Nakamura H, et al. (2003) Osteoprotegerin regulates bone formation through a coupling mechanism with bone resorption. Endocrinology 144: 5441-5449.

5. Cao L, Ding Y. Effects of estrogen and wall-shear stress on rat osteoblats in vitro. J Pract Stomatol 19: 475-478.

6. Kapur S, Baylink DJ, Lau KH (2003) Fluid flow shear stress stimulates human osteoblast proliferation and differentiation through multiple interacting and competing signal transduction pathways. Bone 32: 241-251.

7. Ornitz DM, Marie PJ (2002) FGF signaling pathways in endochondral and intramembranous bone development and human genetic disease. Genes Dev 16: 1446-1465.

8. Marie PJ (2003) Fibroblast growth factor signaling controlling osteoblast differentiation. Gene 316: 23-32.

9. Ornitz DM, Itoh N (2015) The fibroblast growth factor signaling pathway. Wiley Interdiscip Rev Dev Biol 4: 215-266.

10. Goetz R, Mohammadi M (2013) Exploring mechanisms of FGF signalling through the lens of structural biology. Nat Rev Mol Cell Biol 14: 166-180.

11. Goetz R, Dover K, Laezza F, Shtraizent N, Huang X, et al. (2009) Crystal structure of a fibroblast growth factor homologous factor (FHF) defines a conserved surface on FHFS for binding and modulation of voltage-gated sodium channels. J Biol Chem 284: 17883-17896.

12. Schlessinger J, Plotnikov AN, Ibrahimi OA, Eliseenkova AV, Yeh BK, et al. (2000) Crystal structure of a ternary FGF-FGFR-heparin complex reveals a dual role for heparin in FGFR binding and dimerization. Mol Cell 6: 743-750.
13. Plotnikov AN, Hubbard SR, Schlessinger J, Mohammadi M (2000) Crystal structures of two FGF-FGFR complexes reveal the determinants of ligand-receptor specificity. Cell 101: 413-424.

14. Johnson DE, Williams LT (1993) Structural and functional diversity in the FGF receptor multigene family. Adv Cancer Res 60: 1-41.

15. He L, Hristova K (2008) Pathogenic activation of receptor tyrosine kinases in mammalian membranes. J Mol Biol 384: 1130-1142.

16. Ornitz DM (2000) FGFs, heparan sulfate and FGFRs: Complex interactions essential for development. Bioessays 22: 108-112.

17. Matsuo I, Kimura-Yoshida C (2013) Extracellular modulation of Fibroblast Growth Factor signaling through heparan sulfate proteoglycans in mammalian development. Curr Opin Genet Dev 23: 399-407.

18. Belov AA, Mohammadi M (2013) Molecular mechanisms of fibroblast growth factor signaling in physiology and pathology. Cold Spring Harb Perspect Biol 5: 239-249.

19. Kuro-o M (2012) Klotho in health and disease. Curr Opin Nephrol Hypertens 21: 362-368.

20. Kuro-o M (2008) Endocrine FGFs and Klothos: Emerging concepts. Trends Endocrinol Metab 19: 239-245.

21. O'Malley BW (1971) Mechanisms of action of steroid hormones. N Engl J Med 284: 370-377.

22. Turner RT, Riggs BL, Spelsberg TC (1994) Skeletal effects of estrogen. Endocr Rev 15: 275-300.

23. Liu H, Li L, Shi W, Yang J, Wu L, et al. (2016) Progress on the role of estrogen receptor in bone formation and metabolism. Genomics Appl Biol 35: 1656-1661.

24. Kuiper GG, Enmark E, Pelto-Huikko M, Nilsson S, Gustafsson JA (1996) Cloning of a novel receptor expressed in rat prostate and ovary. Proc Natl Acad Sci U S A 93: 5925-5930.

25. Mosselman S, Polman J, Dijkema R (1996) ER beta: Identification and characterization of a novel human estrogen receptor. FEBS Lett 392: 49-53.

26. Wu HY, Zheng P, Jiang G, Liu Y, Nephew KP, et al. (2012) A modulator based regulatory network for ERa signaling pathway. BMC Genomics 13: S6.

27. Tsai YC, Hsu CC, El-Shazly M, Chiang SY, Wu CC (2015) Phytochemicals and estrogen-receptor agonists from the aerial parts of liriope platyphylla. Molecules 20: 6844-6855.

28. Murdoch FE, Gorski J (1991) The role of ligand in estrogen receptor regulation of gene expression. Mol Cell Endocrinol 78: C103-C108.

29. Driscoll MD, Sathya G, Muyan M, Klinge CM, Hilf R, et al. (1998) Sequence requirements for estrogen receptor binding to estrogen response elements. J Biol Chem 273: 29321-29330.

30. Kumar V, Chambon P (1998) The estrogen receptor binds tightly to its responsive element as a ligand-induced homodimer. Cell 55: 145-156.

31. Sundermann EE, Maki PM, Bishop JR (2010) A review of estrogen receptor a gene (ESR1) polymorphisms, mood and cognition. Menopause 17: 874-886.

32. Farooq A (2015) Structural and functional diversity of estrogen receptor ligands. Curr Top Med Chem 15: 1372-1384.

33. Chen WC, Huang ZS, Wei YS, Zhang BY (2010) The research of Rsa and Alu polymorphism in the estrogen receptor- $\beta$ gene associated with Zhuang People centenarians of Guangxi Bama area (in Chinese). Guangdong Med J 31: 1531-1533.

34. Lu SJ, Zhang HQ, Guo CF, Gao QL, Tang MX (2011) The role of estrogen receptor gene ERß in the mouse spinal vertebrae linear growth (in Chinese). China J Modern Med 21: 4518-4523.

35. Gangapuram M, Eyunni S1, Redda KK1 (2014) Synthesis and pharmacological evolution of tetrahydroisoquinolines as anti-breast cancer agents. J Cancer Sci Ther 6: 161-169.

36. Teyssier C, Gallet M, Rabier B, Monfoulet L, Dine J, et al. (2009) Absence of ERR alpha in female mice confers resistance to bone loss induced by age or estrogen-deficiency. PLoS One 4: e7942. 
37. Zallone A (2006) Direct and indirect estrogen actions on osteoblasts and osteoclasts. Ann N Y Acad Sci 1068: 173-179.

38. Albright F. Smith PH (1941) Postmenopausal osteoporosis. Br J Hosp Med (Lond) 38: 2465-2474.

39. Jin CX, Wang YL, Liu SY, Liu DY (2006) The impact of estrogen replacement therapy upon alveolar bone absorption in osteoporosis rats with experimental periodontitis. Journal of Tianjin Medical University 12: 192-195.

40. Song L, Zhao J, Zhang X, Li H, Zhou Y (2013) Icariin induces osteoblast proliferation, differentiation and mineralization through estrogen receptor-mediated ERK and JNK signal activation. Eur J Pharmacol 714: $15-22$.

41. Yang YH, Chen K, Li B, Chen JW, Zheng XF (2013) Estradiol inhibits osteoblast apoptosis via promotion of autophagy through the er-erk-mtor pathway. Apoptosis 18: 1363-1375.

42. Jessop HL, Rawlinson SC, Pitsillides AA, Lanyon LE (2002) Mechanical strain and fluid movement both activate extracellular regulated kinase (ERK) in osteoblast-like cells but via different signaling pathways. Bone 31: 186-194.

43. Adachi T, Sato K, Tomita Y (2003) Directional dependence of osteoblastic calcium response to mechanical stimuli. Biomech Model Mechanobiol 2: 73-82.

44. Chen DB, Bird IM, Zheng J, Magness RR (2004) Membrane estrogen receptor-dependent extracellular signal-regulated kinase pathway mediates acute activation of endothelial nitric oxide synthase by estrogen in uterine artery endothelial cells. Endocrinology 145: 113-125.

45. Salter DM, Robb JE, Wright MO (1997) Electrophysiological responses of human bone cells to mechanical stimulation: Evidence for specific integrin function in mechanotransduction. J Bone Miner Res 12: 1133-1141.

46. Francis GS (2001) Pathophysiology of chronic heart failure. Am J Med 110: $37-46$.

47. Sunadome K, Yamamoto T, Ebisuya M, Kondoh K, Sehara-Fujisawa A, et al. (2011) ERK5 regulates muscle cell fusion through Klf transcription factors. Dev Cell 20: 192-205.

48. Zhao LG, Chen SL, Teng YJ, An LP, Wang J, et al. (2014) The MEK5/ ERK5 pathway mediates fluid shear stress promoted osteoblast differentiation. Connect Tissue Res 55: 96-102.

49. Wang B, Du T, Wang Y, Yang C, Zhang S, et al. (2011) Focal adhesion kinase signaling pathway is involved in mechanotransduction in mg-63 cells. Biochem Biophys Res Commun 410: 671-676.

50. Li JY, Liu SG, Xiao GN, Mao MY, Zhang XW, et al. (2017) Fibroblast growth factor receptor 1 propagates estrogen and fluid shear stress driven proliferation and differentiation response in mc3t3-e1 cells. Mole Biol 51: 300-312.

51. Sakata T, Wang Y, Halloran BP, Elalieh HZ, Cao J, et al. (2004) Skeletal unloading induces resistance to insulin-like growth factor-I (IGF-I) by inhibiting activation of the IGF-I signaling pathways. J Bone Miner Res 19: 436-446.

52. Mendez P, Garcia-Segura LM (2006) Phosphatidylinositol 3-kinase and glycogen synthase kinase 3 regulate estrogen receptor-mediated transcription in neuronal cells. Endocrinology 147: 3027-3039.

53. Kahlert S, Nuedling S, van Eickels M, Vetter H, Meyer R, et al. (2000) Estrogen receptor alpha rapidly activates the IGF-1 receptor pathway. J Biol Chem 275: 18447-18453.

54. Kido S, Kuriwaka-Kido R, Umino-Miyatani Y, Endo I, Inoue D, et al. (2010) Mechanical stress activates Smad pathway through PKCd to enhance interleukin-11 gene transcription in osteoblasts. PLoS One 5: e13090.

55. Sawakami K, Robling AG, Ai M, Pitner ND, Liu D, et al. (2006) The Wnt co-receptor LRP5 is essential for skeletal mechanotransduction but not for the anabolic bone response to parathyroid hormone treatment. J Biol Chem 281: 23698-23711.

56. Lau KH, Kapur S, Kesavan C, Baylink DJ (2006) Up-regulation of the Wnt, estrogen receptor, insulin-like growth factor-I and bone morphogenetic protein pathways in C57BL/6J osteoblasts as opposed to $\mathrm{C} 3 \mathrm{H} / \mathrm{HeJ}$ osteoblasts in part contributes to the differential anabolic response to fluid shear. J Biol Chem 281: 9576-9588.

57. Jessop HL, Suswillo RF, Rawlinson SC, Zaman G, Lee K, et al. (2004) Osteoblast-like cells from estrogen receptor alpha knockout mice have deficient responses to mechanical strain. J Bone Miner Res 19: 938-946.

58. Kouzmenko AP, Takeyama K, Ito S, Furutani T, Sawatsubashi S, et al. (2004) Wnt/beta-catenin and estrogen signaling converge in vivo. J Biol Chem 279: 40255-402258.

59. Foo C, Frey S, Yang HH, Zellweger R, Filgueira L (2007) Downregulation of beta-catenin and transdifferentiation of human osteoblasts to adipocytes under estrogen deficiency. Gynecol Endocrinol 23: 535-540.

60. Katoh M (2006) Crosstalk of WNT and FGF signaling pathways at GSK3beta to regulate beta-catenin and SNAIL singnaling cascades. Cancer Biol Ther 5: 1059-1064.

61. Zaman G, Cheng MZ, Jessop HL (2000) White R, Lanyon LE. Mechanical strain activates estrogen response elements in bone cells. Bone 27: 233-239.

62. Lee K, Jessop H, Suswillo R, Zaman G, Lanyon L (2003) Endocrinology: Bone adaptation requires oestrogen receptor-alpha. Nature 424: 389.

63. Damien E, Price JS, Lanyon LE (2000) Mechanical strain stimulates osteoblast proliferation through the estrogen receptor in males as well as females. J Bone Miner Res 15: 2169-2177.

64. Jessop HL, Sjöberg M, Cheng MZ, Zaman G, Wheeler-Jones CP, et al. (2001) Mechanical strain and estrogen activate estrogen receptor a in bone cells. JBMR 16: 1045-1055.

65. Lanyon LE, Armstrong VJ, Saxon LK, Sunters A,Sugiyama T (2007) Estrogen receptors critically regulate bones' adaptive responses to loading. Clin Rev Bone Miner Metab 5: 234-248.

66. Joldersma M, Klein-Nulend J, Oleksik AM, Heyligers IC, Burger EH (2001) Estrogen enhances mechanical stress-induced prostagland in production by bone cells from elderly women. Am J Physiol Endocrinol Metab 280: 436-442.

67. Bakker AD, Klein-Nulend J, Tanck E, Albers GH, Lips P, et al. (2005) Additive effects of estrogen and mechanical stress on nitric oxide and prostaglandin E2 production by bone cells from osteoprotic dornors. Osteoporosis Int 16: 983-989.

68. Yeh CR, Chiu JJ, Lee CI, Lee PL, Shih YT, et al. (2010) Estrogen augments shear stress-induced signaling and gene expression in osteoblast-like cells via estrogen receptor-mediated expression of ß1-integrin. J Bone Miner Res 25: 627.

69. Eswarakumar VP, Lax I, Schlessinger J (2005) Cellular signaling by fibroblast growth factor receptors. Cytokine Growth Factor Rev 16: 139-149.

70. Choi SC, Kim SJ, Choi JH, Park CY, Shim WJ, et al. (2008) Fibroblast growth factor- 2 and 4 promote the proliferation of bone marrow mesenchymal stem cells by the activation of the PI3K-Akt and ERK1/2 signaling pathways. Stem Cells Dev 17: 725-736.

71. Park J, Park OJ, Yoon WJ, Kim HJ, Choi KY, et al. (2012) Functional characterization of a novel FGFR2 mutation, E731K, in craniosynostosis. J Cell Biochem 113: 457-464.

72. Kim HJ, Kim JH, Bae SC, Choi JY, Ryoo HM (2003) The protein kinase C pathway plays a central role in the fibroblast growth factor-stimulated expression and transactivation activity of Runx2. J Biol Chem 278: 319-326.

73. Raucci A, Bellosta P, Grassi R, Basilico C, Mansukhani A (2008) Osteoblast proliferation or differentiation is regulated by relative strengths of opposing signaling pathways. J Cell Physiol 215: 442-451.

74. Dudka AA, Sweet SM, Heath JK (2010) Signal transducers and activators of transcription-3 binding to the fibroblast growth factor receptor is activated by receptor amplification. Cancer Res 70: 3391-3401.

75. Raman M, Chen W, Cobb MH (2007) Differential regulation and properties of MAPKs. Oncogene 26: 3100-3112.

76. Krishna M, Narang H (2008) The complexity of mitogen-activated protein kinases (MAPKs) made simple. Cell Mol Life Sci 65: 3525-3544. 
77. Wang X, Tournier C (2006) Regulation of cellular functions by the ERK5 signalling pathway. Cell Signal 18: 753-760.

78. Yang SH, Sharrocks AD, Whitmarsh AJ (2013) MAP kinase signalling cascades and transcriptional regulation. Gene 513: 1-13.

79. Kim BG, Kim HJ, Park HJ, Kim YJ, Yoon WJ, et al, (2006) Runx2 phosphorylation induced by fibroblast growth factor-2/protein kinase $\mathrm{C}$ pathways. Proteomics 6: 1166-1174.

80. Miraoui H, Oudina K, Petite H, Tanimoto Y, Moriyama K, et al. (2009) Fibroblast growth factor receptor 2 promotes osteogenic differentiation in mesenchymal cells via ERK1/2 and protein kinase C signaling. J Biol Chem 284: 4897-4904.

81. Jeon E, Yun YR, Kang W, Lee S, Koh YH, et al. (2012) Investigating the role of FGF18 in the cultivation and osteogenic differentiation of mesenchymal stem cells. PLoS One 7: e43982.

82. Hamidouche Z, Fromigue O, Nuber U, Vaudin P, Pages JC, et al. (2010) Autocrine fibroblast growth factor 18 mediates dexamethasone-induced osteogenic differentiation of murine mesenchymal stem cells. J Cell Physiol 224: 509-515.

83. Ohbayashi N, Shibayama M, Kurotaki Y, Imanishi M, Fujimori T, et al. (2002) FGF18 is required for normal cell proliferation and differentiation during osteogenesis and chondrogenesis. Genes Dev 16: 870-879.

84. Behr B, Sorkin M, Manu A, Lehnhardt M, Longaker MT, et al. (2011) Fgf-18 is required for osteogenesis but not angiogenesis during long bone repair. Tissue Eng Part A 17: 2061-2069.

85. Martin A, Liu S, David V, Li H, Karydis A, et al. (2011) Bone proteins PHEX and DMP1 regulate fibroblastic growth factor Fgf23 expression in osteocytes through a common pathway involving FGF receptor (FGFR) signaling. FASEB J 25: 2551-2562.

86. Feng JQ, Clinkenbeard EL, Yuan B, White KE, Drezner MK (2013) Osteocyte regulation of phosphate homeostasis and bone mineralization underlies the pathophysiology of the heritable disorders of rickets and osteomalacia. Bone 54: 213-221.

87. Schell U, Hehr A, Feldman GJ, Robin NH, Zackai EH, et al. (1995) Mutations in FGFR1 and FGFR2 cause familial and sporadic Pfeiffer syndrome. Hum Mol Genet 4: 323-328.

88. Eswarakumar VP, Monsonego-Ornan E, Pines M, Antonopoulou I, Morriss-Kay GM, et al. (2002) The IIIc alternative of Fgfr2 is a positive regulator of bone formation. Development 129: 3783-3793.

89. Yu K, Xu J, Liu Z, Sosic D, Shao J, et al. (2003) Conditional inactivation of FGF receptor 2 reveals an essential role for FGF signaling in the regulation of osteoblast function and bone growth. Development 130: 3063-3074.

90. Marie PJ, Coffin JD, Hurley MM (2005) FGF and FGFR signaling in chondrodysplasias and craniosynostosis. J Cell Biochem 96: 888-896.

91. Valverde-Franco G, Liu H, Davidson D, Chai S, Valderrama-Carvajal H, et al. (2004) Defective bone mineralization and osteopenia in young adult FGFR3-/- mice. Hum Mol Genet 13: 271-284.

92. Su N, Sun QD, Li C, Lu XM, Qi HB, et al. (2010) Gain-of-function mutation in FGFR3 in mice leads to decreased bone mass by affecting both osteoblastogenesis and osteoclastogenesis. Hum. Mol Genet 19: 1199-1210.

93. Matsushita T, Wilcox WR, Chan YY, Kawanami A, Bükülmez H et al. (2009) FGFR3 promotes synchondrosis closure and fusion of ossification centers through the MAPK pathway. Hum Mol Genet 18: 227-240.
94. Yu HM, Jerchow B, Sheu TJ, Liu B, Costantini F, et al. (2005) The role of Axin2 in calvarial morphogenesis and craniosynostosis. Development 132: 1995-2005.

95. Liu B, Yu HM, Hsu W (2007) Craniosynostosis caused by Axin2 deficiency is mediated through distinct functions of beta-catenin in proliferation and differentiation. Dev Biol 301: 298-308.

96. Fei Y, Xiao L, Doetschman T, Coffin DJ, Hurley MM (2011) Fibroblast growth factor 2 stimulation of osteoblast differentiation and bone formation is mediated by modulation of the wnt signaling pathway. J Biol Chem 286: 40575-40583.

97. Mansukhani A, Ambrosetti D, Holmes G, Cornivelli L, Basilico C (2005) Sox2 induction by FGF and FGFR2 activating mutations inhibits Wnt signaling and osteoblast differentiation. J Cell Biol 168: 1065-1072.

98. Kratochwil K, Galceran J, Tontsch S, Roth W, Grosschedl R (2002) FGF4, a direct target of LEF1 and Wnt signaling, can rescue the arrest of tooth organogenesis in Lef1 (-/-) mice. Genes Dev 16: 3173-3185.

99. Shimokawa T, Furukawa Y, Sakai M, Li M, Miwa N, et al. (2003) Involvement of the FGF18 gene in colorectal carcinogenesis, as a novel downstream target of the beta-catenin/T-cell factor complex. Cancer Res 63: 6116-6120.

100. Domingos PM, Itasaki N, Jones CM, Mercurio S, Sargent MG, et al. (2001) The Wnt/-cateninpathway posteriorizes neural tissue in Xenopus by an indirect mechanism requiring FGF signaling. Dev Biol 239: $148-160$.

101. Warren SM, Brunet LJ, Harland RM, Economides AN, Longaker MT (2003) The BMP antagonist noggin regulates cranial suture fusion. Nature 422: 625-629.

102. Reinhold MI, Abe M, Kapadia RM, Liao Z, Naski MC (2004) FGF18 represses noggin expression and is induced by calcineurin. J Biol Chem 279: 38209-38219.

103. Fakhry A, Ratisoontorn C, Vedhachalam C, Salhab I, Koyama E, et al. (2005) Effects of FGF-2/-9 in calvarial bone cell cultures: Differentiation stage dependent mitogenic effect, inverse regulation of BMP-2 and noggin and enhancement of osteogenic potential. Bone 36: 254-266.

104. Choi KY, Kim HJ, Lee MH, Kwon TG, Nah HD, et al. (2005) Runx2 regulates FGF2-induced Bmp2 expression during cranial bone development. Dev Dyn 233: 115-121.

105. Nakamura Y, Tensho K, Nakaya H, Nawata M, Okabe T, et al. (2005) Low dose fibroblast growth factor-2 (FGF-2) enhances bone morphogenetic protein-2 (BMP-2)-induced ectopic bone formation in mice. Bone 36: 399-407.

106. Robinson JA, Chatterjee-Kishore M, Yaworsky PJ, Cullen DM, Zhao W, et al. (2006) Wnt/beta-catenin signaling is a normal physiological response to mechanical loading in bone. J Biol Chem 281:31720-31728.

107. Burch ML, Yang SN, Ballinger ML, Getachew R, Osman N, et al. (2010) TGF-beta stimulates biglycan synthesis via p38 and ERK phosphorylation of the linker region of Smad2. Cell Mol Life Sci 67: 2077-2090.

108. Jun JH, Yoon WJ, Seo SB, Woo KM, Kim GS, et al. (2010) BMP2activated Erk/MAP kinase stabilizes Runx2 by increasing p300 levels and histone acetyltransferase activity. J Biol Chem 285: 36410-36419. 